\title{
A Dynamical Origin for Early Mass Segregation in Young Star Clusters
}

\author{
Steve McMillan, ${ }^{1}$ Enrico Vesperini ${ }^{1}$ and Simon Portegies Zwart ${ }^{2}$ \\ ${ }^{1}$ Department of Physics, Drexel University, Philadelphia, PA 19104, USA \\ email: steve@physics.drexel.edu \\ email: vesperin@physics.drexel.edu \\ ${ }^{2}$ Astronomical Institute 'Anton Pannekoek' and Section Computational Science, University of \\ Amsterdam, Kruislaan 403, 1098SJ Amsterdam, the Netherlands \\ email: spz@science.uva.nl
}

\begin{abstract}
Some young star clusters show a degree of mass segregation that is inconsistent with the effects of standard two-body relaxation from an initially unsegregated system without substructure, in virial equilibrium, and it is unclear whether current cluster formation models can account for this degree of initial segregation in clusters of significant mass. We show that mergers of small clumps that are either initially mass segregated, or in which mass segregation can be produced by two-body relaxation before they merge, generically lead to larger systems which inherit the progenitor clumps' segregation. We conclude that clusters formed in this way are naturally mass segregated, accounting for the anomalous observations and suggesting that this process of prompt mass segregation due to initial clumping should be taken into account in models of cluster formation and dynamics.
\end{abstract}

Keywords. stellar dynamics; methods: n-body simulations; stars: formation; globular clusters: general; open clusters and associations: general

\section{Introduction}

Mass segregation has been observed in many old globular clusters (Sosin \& King 1997, Pasquali et al. 2004), consistent with the fact that these systems have relaxation times significantly less than a Hubble time. However, a number of studies show significant mass segregation in clusters having actual ages, as measured by the evolutionary state of their stars, substantially less than the time needed to produce the observed segregation by standard two-body relaxation (Hillenbrand 1997, Hillenbrand \& Hartmann 1998, Fischer et al. 1998, de Grijs et al. 2002, Sirianni et al. 2002, Gouliermis et al. 2004, Stolte et al. 2006). Numerical simulations indicate that dynamical evolution from initially unsegregated systems cannot account for the degree of mass segregation observed in these clusters (e.g. Bonnell \& Davies 1998).

The obvious explanation is that these clusters were born mass segregated, and recent studies do indeed suggest that massive stars form preferentially in the centers of starforming regions (Elmegreen \& Krakowski 2001, Klessen 2001, Bonnell et al. 2001, Stanke et al. 2006, Bonnell \& Bate 2006). The mechanism invoked to explain this primordial mass segregation relies mainly on the higher accretion rate for stars in the centers of young clusters. However, the efficiency of this mechanism is still a matter of debate (Klein \& McKee 2005, Bonnell \& Bate 2006) and, more generally, the processes of massive star formation and feedback remain poorly understood (Krumholz et al. 2005).

We report results from a numerical study exploring dynamical routes to mass segregation during the early stages of cluster formation (McMillan, Vesperini, \& Portegies Zwart 2007). We imagine that stars form in small clumps, which subsequently merge to 
form larger systems (Bonnell, Bate \& Vine 2003; Elmegreen 2006 and references therein). We assume that the clumps are significantly mass segregated at formation, or that they have short enough relaxation times that mass segregation can occur within the merger time scale. In either case, the final clusters inherit the segregation of their progenitor clumps, providing a natural explanation for large systems which are mass segregated yet physically young.

\section{Method and initial conditions}

We adopt initial conditions in which the cluster consists of $N_{c}$ clumps, with centers distributed within a sphere of radius $R_{\text {cluster }}$. The system of clumps is not in virial equilibrium; rather the clump centers are dynamically "cool," with $q_{c}=-T / U<\frac{1}{2}$. Our simulations have $N_{c}=2,4$, and 8 , and explore the evolution of systems having a range of values of several key bulk parameters of the clump system:

- Clumping Ratio. The ratio $\mathcal{R}_{c} \equiv R_{h} / R_{\text {cluster }}$, where $R_{h}$ is the half-mass radius of an individual clump, is a convenient measure of clumping. We concentrate on two sets of runs: "strongly clumped" models, with $\mathcal{R}_{c}=0.013$, and "moderately clumped" clusters with $\mathcal{R}_{c}=0.037$. (The $90 \%$ Lagrangian radius for a clump is $\sim 5 R_{h}$.)

- Virial Ratio. Our clump systems are initially out of equilibrium, with $0 \leqslant q_{c} \lesssim 0.25$.

- Survey of Two-Clump Mergers. The case $N_{c}=2$ gives us greatest control over the parameters of the interaction, and in this case we vary systematically the clump mass ratio $\mu=M_{1} / M_{2}$, the impact parameter $b$, virial ratio $q$, and the clump mass-radius relation $R_{h} \sim M^{\alpha}$. Our survey spans the ranges $\mu=1,2,4 ; b=0, r_{h} ; q_{c}=0,0.1,0.25$; and $\alpha=0, \frac{1}{3}, \frac{1}{2}$.

Individual clumps are modeled as systems of $N \sim 10^{4}$ particles in virial equilibrium, with Plummer density profiles. We neglect initial binaries and concentrate on two stellar mass distributions:

- Two-component mass functions consisting simply of a "heavy" and a "light" component, for conceptual ease.

- Realistic mass functions as defined by Kroupa, Tout, \& Gilmore 1993.

The degree of mass segregation is conveniently quantified by the ratio $f_{\text {seg }}=R_{h} / R_{h}^{(\text {heavy })}$, where $R_{h}$ is the half-mass radius of the entire system and $R_{h}^{(\text {heavy })}$ refers to the heavy component in the two-component case and to stars above $2.5 M_{\odot}$ in the realistic case.

We consider both initially unsegregated clumps and clumps with significant initial mass segregation. Initial mass segregation in the latter case is achieved by letting an unsegregated clump evolve in isolation for long enough for mass segregation to occur by normal two-body relaxation. This mass-segregated system is then used as a template for all clumps in our simulations. This procedure is simply a convenient means of generating a self-consistent system as an initial condition for a mass-segregated clump; our results are insensitive to the precise means by which the segregation comes about.

Our study is based on direct $N$-body simulations using the starlab package (Portegies Zwart et al. 2001; http://www.manybody.org), accelerated by GRAPE-6 specialpurpose hardware (Makino et al. 2003). Throughout, our time unit is the dynamical time scale (Heggie \& Mathieu 1986) of one of the initial unsegregated clumps. In these units, the internal clump relaxation time scale is $t_{r} \sim 0.1 N / \ln N \sim 100$ (for $N=10^{4}$ ); the free-fall time for the cluster is $t_{f f} \sim 0.7 \mathcal{R}_{c}^{-3 / 2} \sim 90$ (450) for $\mathcal{R}_{c}=0.037(0.013)$.

The results of our simulations are presented in more detail by Vesperini, McMillan, \& Portegies Zwart (2008). 


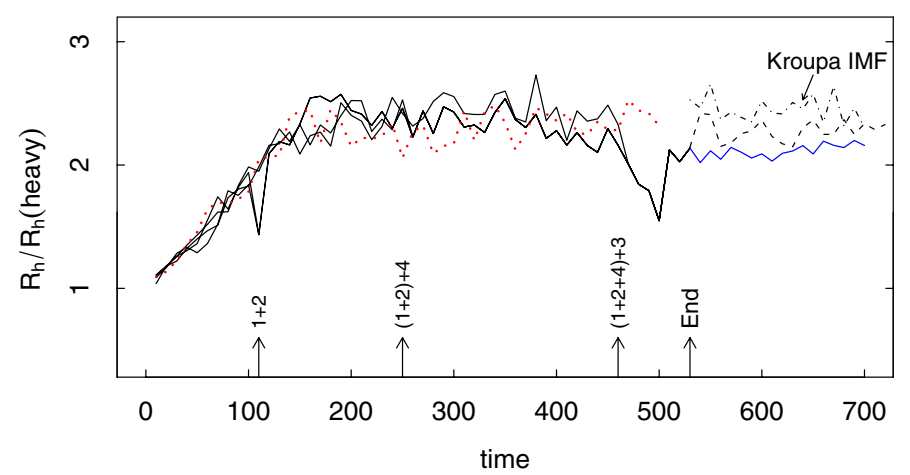

Figure 1. Time evolution of $f_{\text {seg }}$ for an initially unsegregated strongly clumped system. Vertical arrows mark various merging events between the clumps (arbitrarily numbered 1-4); the labels above each arrow indicate the clumps involved in the merger. The solid lines at each stage of the merging process show the evolution of $f_{\text {seg }}$ for the remaining clumps in the cluster. The dotted lines show $f_{\text {seg }}$ for an individual clump evolved in isolation. The dot-dashed line shows the results of a simulation with a Kroupa initial mass function.

\section{Results}

\subsection{Systems with initial mass segregation}

The goal of the initially segregated runs is to establish a connection between the mass segregation of the original clumps and that of the cluster resulting from the merger. We have performed "hierarchical" simulations, in which clumps merge sequentially in a series of two-body encounters, and "cluster" simulations, in which all clumps are followed simultaneously.

In all cases, once the merger is complete, the degree of mass segregation in the final cluster, as measured by $f_{\text {seg }}$, is approximately equal to that in the original clumps-mass segregation is preserved during the merging process. This is consistent with van Albada (1982) and Funato et al. (1992), who found that memory of particles' initial binding energy is not erased during violent relaxation. This result is largely insensitive to any of the structural parameters listed in the previous section, with the sole proviso that, for the clumps to merge into an effectively featureless (smooth) cluster within a few free-fall times, the initial clump system must be relatively cool- $q_{c} \lesssim 0.1$.

Unlike $f_{\text {seg }}$, other bulk properties of the resultant cluster, e.g. central concentration and virial radius, do depend on the properties of the initial clumps - denser clumps tend to produce more concentrated final clusters.

\subsection{Initially unsegregated systems}

We have repeated many of the $N_{c}=4,8$ simulations, without initial mass segregation in the individual clumps. We find that the segregation properties of the end-products are controlled by the ratio $\tau=t_{f f} / t_{\text {seg }}$, representing the degree to which significant internal mass segregation can occur in a global free-fall time. For our choice of system parameters, with $t_{\text {seg }} \sim 0.1 t_{r}, \tau \sim 2$ for moderately clumped initial conditions, and $\tau \sim 10$ for the strongly clumped case, so significant mass segregation is expected within a merger time.

Fig. 1 shows the time evolution of $f_{\text {seg }}$ for an initially unsegregated, strongly clumped $\left(N_{c}=4\right)$ system. It shows the detailed merger history of the original clumps, illustrating how mass segregation proceeds first within the clumps, then within each new merger product, culminating in the final merged cluster. We clearly see internal mass segregation 

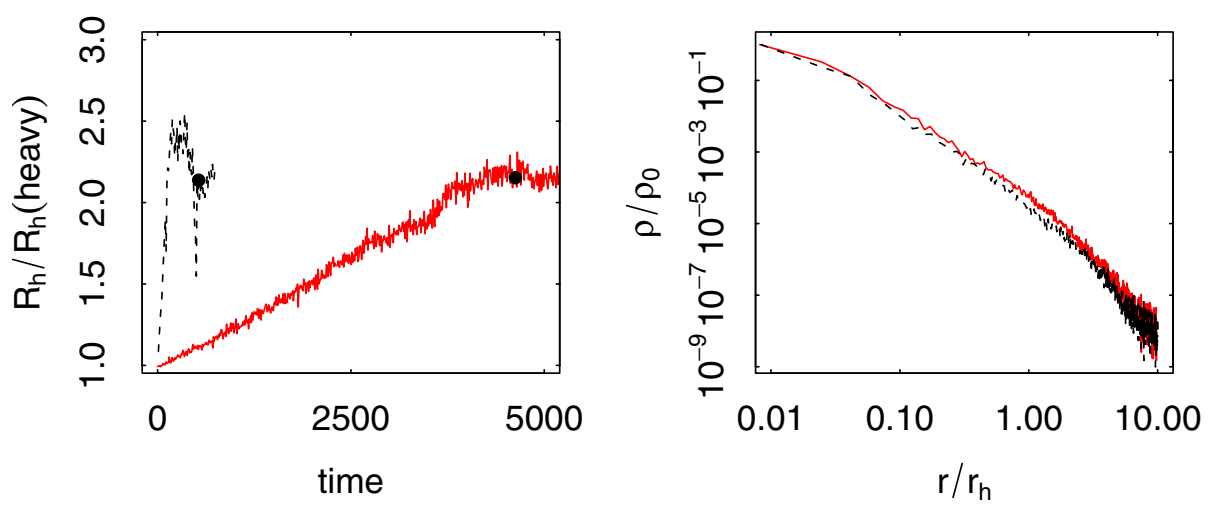

Figure 2. (Left) Time evolution of $f_{\text {seg }}$ for one of the simulations discussed in $\S 4$. The left (dashed) curve began from strongly clumped unsegregated initial conditions with $N_{c}=4$; the right (solid) curve from a single unsegregated Plummer profile. (Right) The density profiles of the two runs at the indicated points are almost indistinguishable.

in the clumps before they merge. The final value of $f_{\text {seg }}$ is comparable to those found in the initially segregated simulations.

As an additional point of comparison, the dot-dashed line in Fig. 1 shows the corresponding ratio for an additional simulation with the same overall parameters but using a mass function from Kroupa et al. (1993), demonstrating that the effect persists when a realistic cluster mass distribution is used.

For this scenario to work, the clumps must have $\tau>1$; for $t_{f f} \sim 1 \mathrm{Myr}$, this implies $t_{r} \lesssim 10$ Myr. We note that 4 (out of 5 ) of the young embedded clusters listed by Baba et al. (2004) have relaxation times between 2 and $10 \mathrm{Myr}$, and the segregated clusters cited in $\S 1$ have relaxation times ranging from $\sim 6 \mathrm{Myr}$ (Orion) to $\sim 40 \mathrm{Myr}$ (NGC 3603). The relaxation time in the final cluster is expected exceed that in a clump by a factor $\approx N_{c}^{1 / 2}\left(0.1 / \mathcal{R}_{c}\right)^{3 / 2}=8.9(43)$ for $N_{c}=4, \mathcal{R}_{c}=0.037(0.013)$. [The " $N$ " in the relaxation time contributes a factor of $N_{c}$, the dynamical time scale contributes $\left(N_{c} / \mathcal{R}_{c}^{3}\right)^{-1 / 2}$, and the numerical factor 0.1 comes from an estimate of the relationship between the final half-mass radius and $R_{\text {cluster }}$, based on energy conservation and the virial theorem.] We conclude that the above condition on $t_{r}$ would be met for even a modest number of clumps or moderate clumping.

\section{Possible dynamical histories of a young segregated cluster}

The end products of the simulations described above are young, yet significantly mass segregated, clusters. Without knowing the actual dynamical history of such a system, one might imagine "observing" one of these simulated clusters to try to reproduce its properties and reconstruct its past dynamical evolution. The traditional way to do this is to perform $N$-body simulations starting from the initial conditions adopted in the vast majority of numerical studies of star cluster evolution - a spherical system with no primordial mass segregation and a Plummer (or King) density profile. We have carried out this experiment, running a simulation starting from a two-component spherical system in virial equilibrium, with 40,000 particles and a Plummer density profile. We refer to this simulation as our "standard" model.

Fig. 2 (left frame) compares the time evolution of $f_{\text {seg }}$ in the standard model with the strongly clumped unsegregated run described in $\S 3$. The standard model is scaled so that, 
at the indicated times, when mass segregation is effectively complete and the degree of mass segregation is similar in each run, the two models have the same half-mass radius. We see that the clumped model achieves "complete" mass segregation much sooner (at least a factor of $\sim 7-10$ faster) than does the standard model.

Furthermore, as shown in the right frame of Fig. 2, the density profiles at the indicated times are very similar. Not shown in the figure is the fact that the median radii corresponding to different mass ranges (for cases with a realistic mass spectrum) are also very similar in the two models. Since the standard model takes much longer than the clumped system age to reproduce the same cluster properties, one might incorrectly conclude from this numerical study that the mass segregation found in this cluster must reflect its initial conditions. However, as we have shown, several possible dynamical histories can lead to similar final systems.

These simulations demonstrate that there are a number of viable evolutionary paths, relying on initial mass segregation in clumpy systems or on multiscale dynamical evolution, that can lead to significant mass segregation in a physically young cluster.

\section{Acknowledgements}

This work was supported in part by NASA grants NNG04GL50G and NNX07AG95G, NSF grant AST-0708299, and by the Royal Netherlands Academy of Arts and Sciences (KNAW).

\section{References}

Bonnell, I. A. \& Davies, M. B 1998, MNRAS, 295, 691

Bonnell, I. A., Clarke, C. J., Bate, M. R., \& Pringle, J. E. 2001, MNRAS, 324, 573

Bonnell, I. A., Bate, M. R., \& Vine S. 2003, MNRAS, 343, 413

Bonnell, I. A. \& Bate, M. R. 2006, MNRAS, 370, 488

de Grijs, R., Gilmore, G. F., Johnson, R. A., \& Mackey, A. D. 2002, MNRAS, 331, 245

Elmegreen, B. \& Krakowski, A. 2001, ApJ, 562, 433

Elmegreen, B. 2006, astro-ph/0605519

Fischer, P., Pryor, C., Murray, S., Mateo, M., \& Richtler, T. 1998, AJ, 331, 592

Funato, Y., Makino, J. \& Ebisuzaki, T. 1992, PASJ, 44, 291

Gouliermis, D., Keller, S. C., Kontizas, M., Kontizas, E., \& Bellas-Velidis, I. 2004, A\&A, 416, 137

Heggie, D. C. \& Mathieu, R. D. 1986, in The Use of Supercomputers in Stellar Dynamics (P. Hut and S. McMillan, eds.; Springer-Verlag, New York)

Hillenbrand, L. A. 1997, AJ, 113, 1733

Hillenbrand, L. A. \& Hartmann L. E. 1998, ApJ, 331, 540.

Klessen R., 2001, ApJ, 556, 837

Kroupa, P., Tout, C. A., \& Gilmore, G. 1993, MNRAS, 262, 545

Krumholz, M. R., Klein, R. I., \& McKee, C. F. 2005, Nature, 438, 332

Makino, J., Fukushige, T., Koga, M., \& Namura, K. 2003, PASJ, 55, 1163

McMillan, S. L. W., Vesperini, E., \& Portegies Zwart, S. F.2007, ApJ, 655, 45

Pasquali, A., De Marchi, G., Pulone, L., \& Brigas, M. S. 2004, A\&A, 428, 469

Portegies, Zwart, S. F., McMillan, S. L. W., Hut, P., \& Makino, J. 2001, MNRAS, 321, 199

Sirianni, M., Nota, A., De Marchi, G., Leitherer, C., \& Clampin, M. 2002, ApJ, 579, 275

Sosin, C., \& King, I. R. 1997, AJ, 113, 1328

Stanke, T., Smith, M. D., Gredel, R., \& Khanzadyan, T. 2006, A\&A, 447, 609

Stolte, A., Brandner, W., Brandl, B., \& Zinnecker H. 2006, AJ, 132, 253

van Albada, T. S. 1982, MNRAS, 201, 939

Vesperini, E., McMillan, S. L. W., \& Portegies Zwart, S. F., 2008, in preparation 